\section{Federation University ResearchOnline}

https://researchonline.federation.edu.au

Copyright Notice

This is the peer-reviewed version of the following article:

Nguyen, L., \& Miro, J. V. (2020). Efficient Evaluation of Remaining Wall Thickness in Corroded Water Pipes Using Pulsed Eddy Current Data. IEEE Sensors Journal, 20(23), 14465-14473.

https://doi.org/10.1109/JSEN.2020.3007868

Copyright (C) 2020 IEEE. Personal use of this material is permitted. Permission from IEEE must be obtained for all other uses, in any current or future media, including reprinting/republishing this material for advertising or promotional purposes, creating new collective works, for resale or redistribution to servers or lists, or reuse of any copyrighted component of this work in other works. 


\title{
Efficient Evaluation of Remaining Wall Thickness in Corroded Water Pipes using Pulsed Eddy Current Data
}

\author{
Linh Nguyen, Member, IEEE, Jaime Valls Miro, Member, IEEE
}

\begin{abstract}
In order to analyse failures of an ageing water pipe, some methods such as the loss-of-section require remaining wall thickness (RWT) along the pipe to be fully known, which can be measured by the magnetism based non-destructive evaluation sensors though they are practically slow due to the magnetic penetrating process. That is, fully measuring RWT at every location in a water pipe is not really practical if RWT inspection causes disruption of water supply to customers. Thus, this paper proposes a new data prediction approach that can increase amount of RWT data of a corroded water pipe collected in a given period of time by only measuring RWT on a part (e.g. 20\%) of the total pipe surface area and then employing the measurements to predict RWT at unmeasured area. It is proposed to utilize a marginal distribution to convert the non-Gaussian RWT measurements to the standard normally distributed data, which can then be input into a 3-dimensional Gaussian process model for efficiently predicting RWT at unmeasured locations on the pipe. The proposed approach was implemented in two real-life in-service pipes, and the obtained results demonstrate its practicality.
\end{abstract}

Index Terms-Water pipes, cast iron pipes, remaining wall thickness, nondestructive testing/evaluation, marginal distribution, Gaussian process.

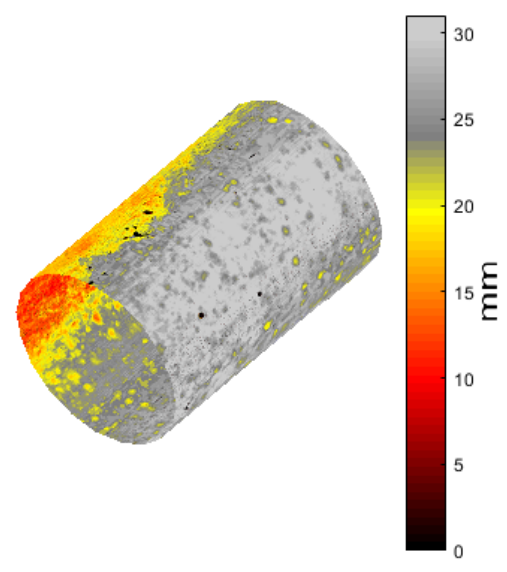

\section{INTRODUCTION}

Studies have shown that about 70 percent of the total global water pipes are buried underground, and most of these pipes have been in service for more than a century [1]-[3]. Due to their critically ageing conditions, these water pipes' failures have been frequently occurring, which cause severe implications for not only sustainability and efficacy of water services but also the living conditions of civilians in surrounding areas including property damages, traffic accidents and obstruction and water supply disruption [4]. Statistically, average loss caused by large diameter water pipe failures usually exceeds US\$500,000 [5]. In order to prevent these severities to communities, assessing conditions of a critical water pipe is highly expected [1]. Hence, some methods such as the fracture mechanics and loss-of-section [6]-[11] have been proposed for computing residual strength or likely failure of the pipe. In contrast to the fracture mechanics approach [8], [10], the loss-of-section technique [11] requires remaining thickness of a pipe wall to be known. In equivalent words, remaining wall thickness (RWT) of a water pipe is required to be measured before its failure analysis can be conducted.

"This work is an outcome from the Operationalisation project funded by Sydney Water Corporation."

L. Nguyen is with the School of Engineering, Information Technology and Physical Sciences, Federation University Australia, VIC 3842, Australia (e-mail: I.nguyen@federation.edu.au).

J. Valls Miro is with Faculty of Engineering and Information Technology, University of Technology Sydney, NSW 2007, Australia (e-mail: jaime.vallsmiro@uts.edu.au).
An example of RWT of a $600 \mathrm{~mm}$ diameter water pipe section is demonstrated in the graphical abstract.

For measuring RWT of a water pipe, which is still under service, many non-destructive testing (NDT) or evaluation (NDE) approaches have been proposed in water industries [12]. Nevertheless, most of those proposed NDT/NDE techniques are based on derivation of magnetism. For instance, methods of the eddy current, pulsed eddy current (PEC) and remote field eddy current measure the induced voltage caused by the eddy current in the pipe material after they generate the magnetic field to penetrate through the pipe wall. Moreover, the magnetic flux leakage technique is required to get the pipe material magnetized before measurements can be obtained. That is, measuring RWT at a particular point on a water pipe by the magnetism based NDE/NDT approaches is practically and inevitably slow [13]-[15].

More importantly, one of the challenging issues when measuring RWT by the eddy current based approaches from inside of a water pipe in the dewatered condition is how to minimize disruption of supplying water to customers. In particular, as discussed in our previous work [5], a robot with mounted sensors is only allowed to collect RWT data of a water pipe when a pipe break occurs. That is, RWT measurements can be collected within a very short period of time between a break occurring and repair taking place. More importantly, the collected RWT information allows a water utility to make necessary decisions to prevent a water pipe from repeated failures in the same geographic area and reduce maintenance 


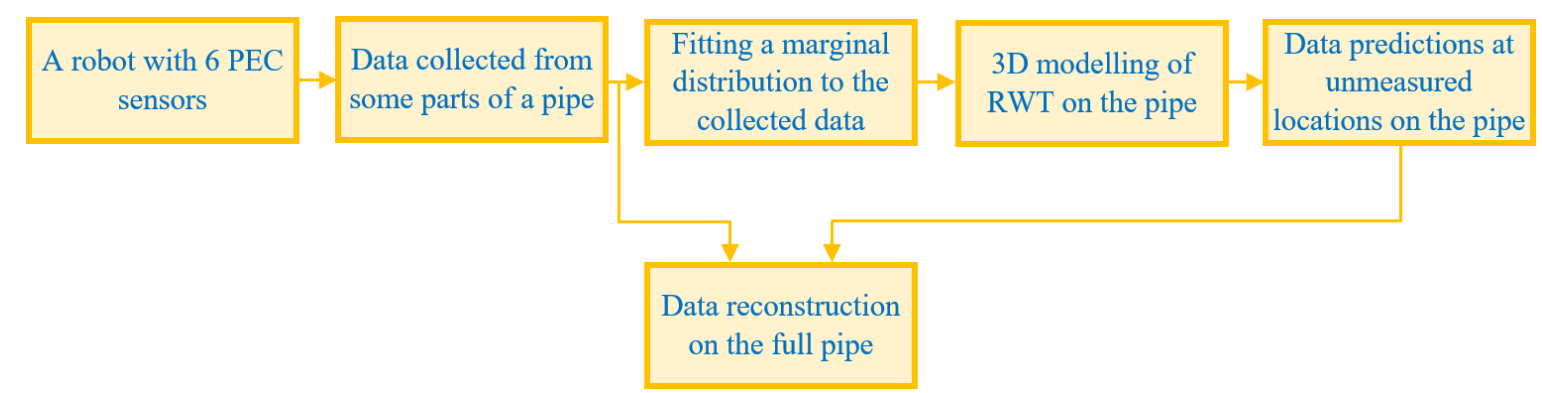

Fig. 1: An efficient data prediction approach in predicting remaining wall thickness of corroded water pipes.

costs by pinpointing and only replacing vulnerable sections of the pipe. Therefore, given a short period of time, we expect to have more RWT data so that the longer pipe sections can be evaluated.

To address the aforementioned problem of the slow measurement, in this work we propose a new data prediction approach where we exploit our previously optimized robot and sensor array system [5] to only measure RWT on a part (e.g. $20 \%$ ) of the total pipe surface area. The RWT measurements are utilized to develop a model of the RWT values in the whole pipeline. More interestingly, the learned model is then used to predict RWT at any unmeasured locations along the pipeline in order to fully reconstruct RWT data on the whole water main. Thus, the main contributions of the paper are summarized as follows.

- A data prediction approach is proposed to predict RWT data at any unmeasured positions along a corroded water pipe given a limited number of RWT measurements collected on a part of the same pipe in a given period of time, particularly when NDE/NDT sensors are deployed in a pipe within a short period of time between a pipe failure/break occurring and repair taking place.

- It is presented how to fit a marginal distribution to the RWT measurements and convert them to the standard normally distributed data to input into a 3-dimensional (3D) Gaussian process (GP) model for effectively predicting RWT at unmeasured locations on a pipe.

- The proposed approach has been experimentally evaluated in the real-life water pipes, and the obtained results promisingly demonstrate its practicality.

It is noted that only RWT of a corroded water pipe is considered in this work and other defects of the pipe are beyond the scope of the paper.

The remaining of the paper is arranged as follows. Overview of the proposed data prediction approach is introduced in Section II. Section III presents a marginal distribution of RWT at a particular point along a pipe before a 3D model of RWT in the whole pipeline is formulated in Section IV. Reconstructing RWT data including measurements and predictions on the whole water main is then discussed in Section V. Section VI delineates the experimental evaluation of the proposed method on the realistic water pipes before the conclusions are drawn in Section VII.

\section{Overview of Proposed Data Prediction APPROACH}

As demonstrated in Fig. 1, a new data prediction approach is proposed, which exploits a robot carrying NDE sensors to measure RWT only at some parts of a pipe wall. A 3D model of RWT along the pipeline is established after the limited number of the RWT measurements are converted to the standard normally distributed data by its corresponding marginal distribution; and RWT data at unmeasured locations are then fully reconstructed from the model.

\section{A. Introduction to Robot and Sensor Array}

For the demonstration purpose, in this work we employ our previously optimized robot and PEC sensor array system [5], [14], [16], [17] to get RWT measurements on a corroded cast iron pipe. The robot is exploited to carry six PEC sensors that are spatially equidistant in circumference as illustrated in Fig. 2a. In the experiments, the robot is deployed inside a dewatered water pipe to scan its internal surface as shown in Fig. 2b. It is noted that the robot can be easily extended to any other corroded water pipes with different pipe wall materials given the properly changed sensors. The PEC sensor allows us to effectively measure RWT of a corroded cast iron water pipe though lift-off, a possible layer between the sensor and pipe material, can be up to $25 \mathrm{~mm}$ [5]. In literature there are rich studies of the lift-off robustness or invariance on eddy current sensor performances; interested readers are referred to the works [18]-[20].

It is also noted that in this work we utilize our previously developed PEC sensors for cast iron water pipes to verify the proposed approach. Nonetheless, discussion on the PEC sensor information including quality of sensor measurements or lift-off influence on sensor signals is beyond our work. More importantly, the proposed method is independent from quality of sensor measurements. In principle, it can similarly be applied for different RWT data obtained by different PEC sensors. In other words, the proposed approach takes a limited number of RWT measurements to learn a model and then employs the learned model to predict RWT at unmeasured location on the same pipe. The prediction of RWT at one location is expected to be more or less similar to the RWT measurement if collected at the same location [21], which allows users to reduce a number of physical measurements or increase RWT data in a given period of time. Our approach 


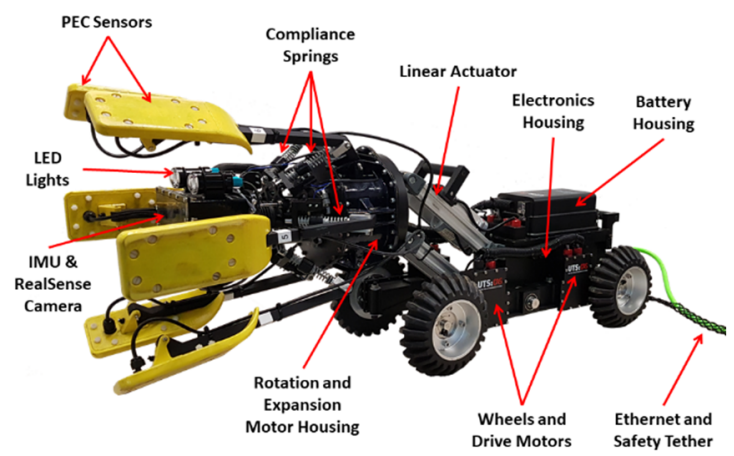

(a)

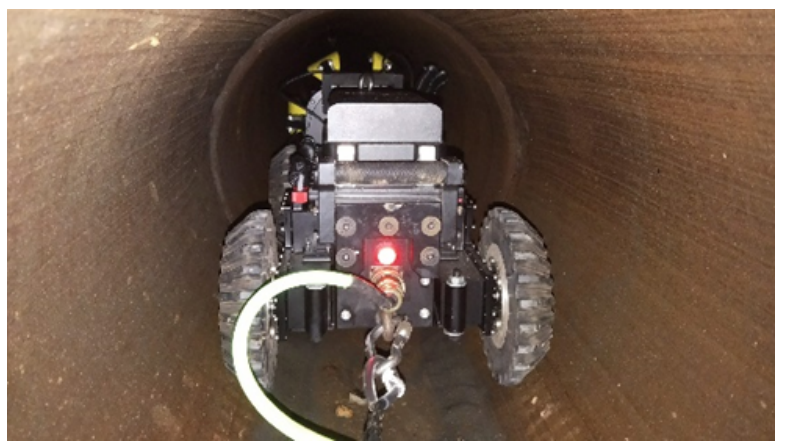

(b)

Fig. 2: (a) A robot with 6 PEC sensors to partly measure RWT of a corroded water pipe. (b) The robot deployment to scan the internal surface of a realistic corroded water pipe in Sydney, Australia.

can also be used for any type of pipes as long as there exists spatial correlation in the sensor measurements.

\section{B. Predictions at Unmeasured Locations}

In order to predict or estimate RWT at the locations along the pipeline where the robot could not inspect due to time constraint or expectation of having the longer corroded water pipe trunk to be evaluated, a 3D Gaussian process (GP) model [21] of RWT in the whole pipe is required to be learned from the RWT measurements collected at the vicinity. Nonetheless, due to the non-Gaussian property of the raw RWT measurements [22], the GP model cannot take them directly. As a result, this work proposes to exploit a marginal distribution to marginalize RWT at a particular point on the corroded water pipe and then convert the RWT measurements to the standard normally distributed data that suits the input of the GP model. Details of the marginal distribution, 3D GP model and data reconstruction at unmeasured locations will be discussed in the following sections.

\section{Marginal Distributions of Measurements}

In the context of statistics, a marginal distribution of a set of measurements is the probability distribution of a variable presenting for them. For the RWT inspection, the marginal distribution can be used to present the probability distribution of RWT data at a particular location on the water pipe. Some previous works have been discussing about the marginal distribution for the pipe thickness; for instance, the authors in [23], [24] proposed to exploit the extreme value (EV) distributions including Weibull and Gumbel to marginally present RWT of a pipe, while in our previous work [25] we discussed about using the Gaussian mixture (GM) distribution to statistically marginalize RWT data that has very left-skewed probability distribution.

\section{A. Extreme Value Marginal Distribution}

In the water pipe failure analysis methods [11], accurately quantifying RWT at the low range (e.g. less than 50 percent of the nominal thickness) is paramount importance. That is the reason why the EV distributions are chosen to marginally present RWT data so that the small RWT measurements can be more accurately modelled. The cumulative distribution function $(\mathrm{CDF})$ of the EV distributions can be specified by [24]

$$
F(t)=\exp \left\{-\left[1+\xi\left(\frac{t-\mu}{\sigma}\right)\right]^{-\frac{1}{\xi}}\right\}
$$

where $t$ is the RWT variable, $\mu$ is the location parameter, $\sigma$ is the scale parameter and $\xi$ is the shape parameter. Given the advantage of the shape parameter, the EV distributions can be fit to any skewed data. It is noted that depending on the shape parameter $\xi$, the CDF (1) can be presented for the Weibull or Gumbel distribution.

\section{B. Gaussian Mixture Marginal Distribution}

Though in theory the EV distributions can approximately marginalize the skewed data, in cases where the probability density distribution (PDF) of the measurements has very long, skewed and tiny left tail as demonstrated in Fig. 4b, the EV distributions cannot fit the data well. In that case, a marginal distribution based on the GM was proposed [25]. The PDF of the GM distribution can be given as follows.

$$
p(t)=\sum_{i=1}^{N} \omega_{i} \mathcal{N}\left(t \mid m_{i}, s_{i}\right),
$$

where $t$ is the RWT variable, $N$ is the number of the components while $m_{i}$ and $s_{i}$ are the mean and standard deviation of the $i^{t h}$ Gaussian component. $\omega_{i}$ is the component weight for the corresponding $i^{t h}$ Gaussian component, where $\sum_{i=1}^{N} \omega_{i}=$ 1. And $\mathcal{N}(\cdot)$ is the Gaussian distribution operator. Since the GM distribution possesses multiple Gaussian components, it is expected that each Gaussian distribution can present a subset of a range of the RWT values. And the number of the Gaussian components needed for a particular RWT dataset can be obtained by computing the Akaike information criterion (AIC), which evaluates the quality of the fitting model.

In other words, if $N_{\max }$ is the maximum number of the Gaussian components in the GM distribution, which is assumed for a particular RWT data set, we compute the $\mathrm{AIC}_{N}$ for the GM distribution with the $N$ Gaussian components, $N=1 \div N_{\max }$. The optimal $N$ corresponds to the minimum $\mathrm{AIC}_{N}$. 


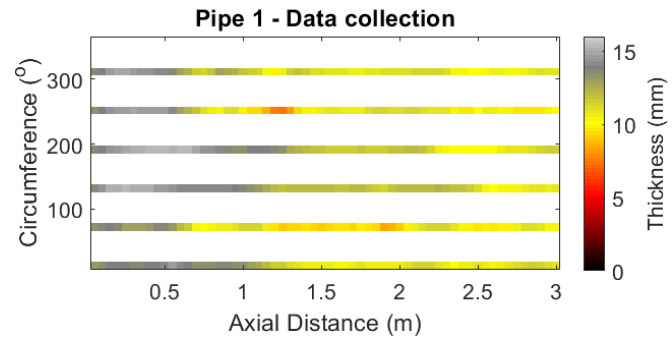

(a)

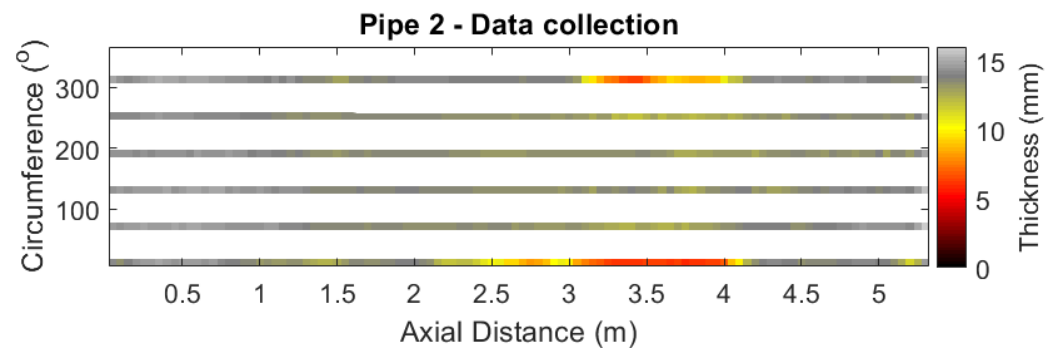

(b)

Fig. 3: Examples of RWT measurements collected on the two different corroded water pipes. There is no data collected in the white areas.

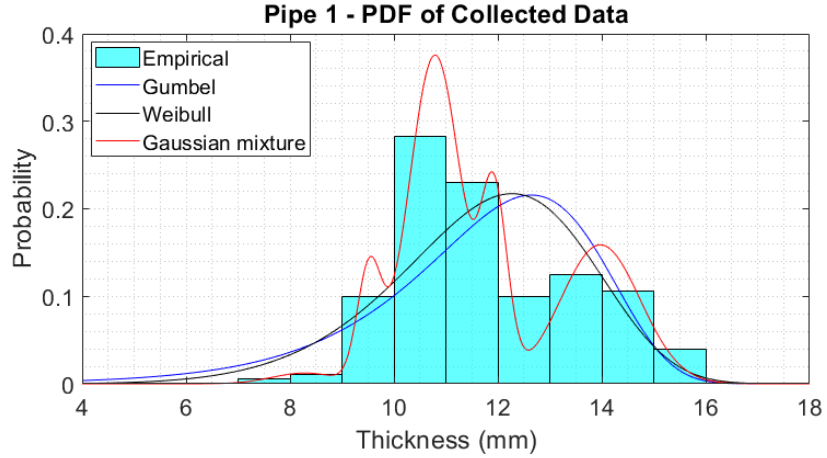

(a)

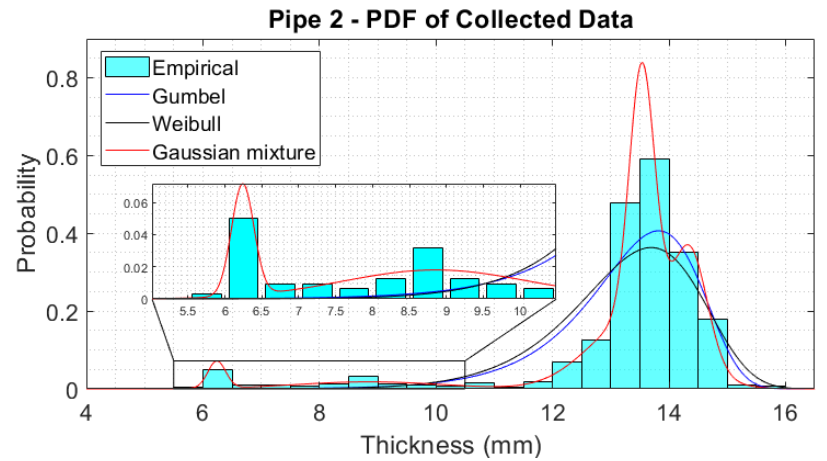

(b)

Fig. 4: Probability density functions (PDFs) of the collected RWT data in the first tests of the two corroded water pipes as shown in Fig. 3, respectively.

In order to demonstrate how the aforementioned marginal distributions fit RWT measurements, we implemented those marginal distributions in the realistic data sets as shown in Fig. 3. The measurements were gathered by the robot as discussed in Section II when it was deployed into two different corroded water pipes. There were six lines along in each of those two pipes were scanned by the six PEC sensors and the obtained RWT measurements are represented by the colors in Fig. 3.

Three marginal distributions including Gumbel, Weibull and GM were fitted to the collected RWT measurements of the two corroded water pipes, respectively; and, their PDFs against the empirical data are shown in Fig. 4. It is noted that the optimal numbers of the Gaussian components for the GM distributions of the RWT observations on the two pipes are 8 and 5 , respectively.

It can be clearly seen that the RWT measurements of the pipe 1 has a very short left-skewed tail as pictured in Fig. 4a, which leads to quite similar fittings of the marginal Gumbel, Weibull and GM distributions in this area. In contrast, the RWT measurements of the pipe 2 have a very long, skewed and tiny left tail as demonstrated in Fig. 4b, where the GM distribution shows its better fitting than its two other counterparts.

In order to numerically compare among those distributions to see how fit to the measurements they are, we computed the AIC values for the corresponding marginal distributions in both the examined water pipes and the obtained results are summarized in Table I. It can be seen that the AIC results in the second column of Table I consolidate the similarity of
TABLE I: AIC COMPARISONS AMONG MARGINAL DISTRIBUTIONS

\begin{tabular}{|l|l|l|}
\hline Marginal distribution & Pipe 1 & Pipe 2 \\
\hline Gumbel & 1478 & 2013 \\
Weibull & 1438 & 2160 \\
Gaussian mixture & 1314 & 1627 \\
\hline
\end{tabular}

three distributions in fitting to the pipe 1 data set. Nevertheless, the AIC results in the third column of Table I clarify the outperformance of the GM distribution in fitting the pipe 2 RWT measurements as compared with the other two.

More importantly, as mentioned in Section II-B, the use of the marginal distribution is to convert the non-Gaussian RWT measurements to the standard normally distributed data to input into the 3D GP model. To that end, it is proposed to compute the inverse of the standard normal CDF as follows,

$$
t^{s}=F^{-1}(p)
$$

where $F(p)$ is the CDF of the marginal distribution, evaluated at the probability value $p$ and $t^{s}$ is the inverse of standard normal CDF.

\section{3D MODEL OF RWT ON THE PIPE}

It can be clearly seen in the graphical abstract that the RWT data collected on a corroded water pipe is cylindrically presented. To model this data, most of the relevant works proposed to unwrap the 3D RWT into 2-dimensional (2D) data. For instance, the authors in [26], [27] proposed 
to simulate the RWT model by using the finite element analysis technique. Though the proposed model has high computational complexity, it is not able to infer RWT at uninspected locations. In the work [25], we employed a 2D GP model to present the collected RWT data after they are unwrapped into 2D. To incorporate the periodicity of the data along the pipe circumference into the model, we exploited a periodic function [28] to tweak the covariance function of the GP model. Although the model can be utilized to predict RWT at unmeasured positions, the generic periodic function inherently calculates the periodicity in both circumferential and longitudinal directions. Nonetheless, there is no existence of periodicity along the longitudinal direction, this presentation is redundant. Therefore, in a recent work [21], we proposed a 3D GP model for RWT in a corroded water pipe. By transforming the cylindrical coordinates on the pipe to the 3D Cartesian coordinates, it is not required to employ the periodic function, but the periodicity of RWT along the circumferential direction of the pipe is naturally presented in the 3D model.

Though a location of every RWT value on the pipe has three cylindrical coordinates including angular coordinate, radial distance and height, which can be used in the 3D GP model, in cases where the radial distance is constant or approximately constant, the length scale hyperparameter of the 3D GP model presenting for this quantity is almost zero. On the other hand, when two locations with similar radial distance and height but their angular coordinates of $1^{\circ}$ and $360^{\circ}$, respectively, are clearly adjacent in the circumference, the GP model still considers these two locations to be faraway from each other. These issues can be addressed by the use of transformation of the $3 \mathrm{D}$ cylindrical coordinates to the 3D Cartesian coordinates as follows.

$$
l=(x, y, z)=(\rho \cos \theta, \rho \sin \theta, z)
$$

where $l=(x, y, z)$ is the 3D Cartesian coordinates. $\rho, \theta$ and $z$ are the radial distance, angular coordinate and height in the cylindrical coordinate, respectively. It is noticed that this transformation is generic where the radial distance may not be constant.

If we consider $t_{i}^{s}$ as the corresponding RWT value measured at the location $l_{i}$, synthesizing all the $n$ corresponding RWT observations $\boldsymbol{t}^{s}=\left(t_{1}^{s}, t_{2}^{s}, \cdots, t_{n}^{s}\right)^{T} \in \mathbb{R}^{n}$ at the locations $\boldsymbol{l}=\left(l_{1}^{T}, l_{2}^{T}, \cdots, l_{n}^{T}\right)^{T} \in \mathbb{R}^{n \times 3}$ on a given water pipe can be presented by

$$
\boldsymbol{t}^{s}(\boldsymbol{l})=\boldsymbol{v}(\boldsymbol{l})+\varepsilon,
$$

where $\boldsymbol{v}=\left(v_{1}, v_{2}, \cdots, v_{n}\right)^{T} \in \mathbb{R}^{n}$ are the latent variables at l. $\varepsilon=\left(\epsilon_{1}, \epsilon_{2}, \cdots \epsilon_{n}\right) \in \mathbb{R}^{n}$, where $\epsilon_{i}$ is a measurement noise of the $i^{\text {th }}$ RWT measurement, which has a zero mean and an unknown variance $\sigma_{n}^{2}$.

If we model the variables $v$ by the GP, where its mean is the constant average of all the marginally converted but standard normally distributed RWT data as discussed in Section III. In theory, this mean should be zero, but in practice it is approximately zero. Since the corrosion processes in soil where the pipe has been buried are directional [29], the processes of the corrosion on the pipe behave quite similarly. That is, the spatial correlations of the corrosion on the pipe in the two circumferential and longitudinal directions, respectively, are different. Therefore, regarding the covariance function for the GP model, in this work, we propose to exploit the automatic relevance determination Matern kernel [28] as follows,

$$
\operatorname{cov}\left(l_{i}, l_{j} \mid \Theta\right)=\sigma^{2}(1+\sqrt{3} d) \exp (-\sqrt{3} d),
$$

where $\operatorname{cov}\left(l_{i}, l_{j} \mid \Theta\right)$ is the dependence between the two RWT variables at locations $l_{i}$ and $l_{j} . \sigma$ is the standard deviation of the RWT measurements, while

$$
d=\sqrt{\sum_{k=1}^{2} \frac{\left(l_{i}-l_{j}\right)^{T}\left(l_{i}-l_{j}\right)}{\nu_{k}^{2}}},
$$

where $\nu_{k}$ is the length scale hyperparameter of the GP model in the $k$ direction, $k=1,2 . \Theta=\left(\sigma, \nu_{1}, \nu_{2}, \sigma_{n}\right)$ is a set of the hyperparameters. These hyperparameters can be estimated by the use of the maximum likelihood method [30] based on all the RWT measurements.

\section{Full Pipe RWT data Reconstruction}

If the robot conducted the inspection on the pipe and collected RWT measurements at the locations $l \in \mathbb{R}^{n \times 3}$, then the standard normally distributed RWT values at the $m$ unmeasured locations $l^{*} \in \mathbb{R}^{m \times 3}$ can be obtained by computing the posterior distribution of the standard normally distributed RWT values at those unmeasured locations, where its mean mean $_{l^{*}}$ and covariances $\Sigma_{l^{*}}$ are as follows,

$$
\begin{gathered}
\operatorname{mean}_{\boldsymbol{l}^{*}} \mid \boldsymbol{t}^{s}(\boldsymbol{l})=\mu\left(\boldsymbol{l}^{*}\right)+\Sigma_{l \boldsymbol{l}^{*}}^{T}\left(\Sigma_{\boldsymbol{l l}}+\sigma_{n}^{2} I\right)^{-1}\left(\boldsymbol{t}^{s}(\boldsymbol{l})-\mu(\boldsymbol{l})\right) \\
\Sigma_{\boldsymbol{l}^{*}} \mid \boldsymbol{t}^{s}(\boldsymbol{l})=\Sigma_{\boldsymbol{l}^{*} \boldsymbol{l}^{*}}-\Sigma_{l l^{*}}^{T}\left(\Sigma_{\boldsymbol{l l}}+\sigma_{n}^{2} I\right)^{-1} \Sigma_{\boldsymbol{l l ^ { * }}},
\end{gathered}
$$

where $\mu\left(\boldsymbol{l}^{*}\right)(\mu(\boldsymbol{l}))$ and $\Sigma_{l^{*} l^{*}}\left(\Sigma_{l l}\right)$ are a $m(n)$ standard normally distributed RWT mean vector and a $m \times m(n \times n)$ covariance matrix at the locations $\boldsymbol{l}^{*}(\boldsymbol{l})$, respectively. $\Sigma_{\boldsymbol{l} \boldsymbol{l}^{*}}$ is a $n \times m$ covariance matrix comprising the correlations between any two the RWT values at locations $l^{*}$ and $l$ while $I$ is a $n \times n$ identity matrix. The elements of those covariance matrices can be calculated by using (6).

It is noted that in Section III we discussed the proposition of the marginal distribution to convert the non-Gaussian realistic RWT measurements to the standard normally distributed RWT values for learning the 3D GP model and then conducting the predictions of the standard normally distributed RWT values at unmeasured locations, as demonstrated in equations (8) and (9). The final step in the inference procedure is to conduct the inversion of the standard normally distributed RWT predictions into the approximation of the realistic RWT values at unmeasured positions. This can be easily done by the use of the parameters of the marginal distributions as introduced in Section III.

By combining the RWT measurements and predictions at their corresponding locations, the fully reconstructed RWT on the full pipe can be presented. 


\section{EXPERIMENTAL RESULTS AND Discussions}

In order to evaluate how the proposed approach performs in reality, we conducted the experiments in the real-life in-service water pipes in the water network of Sydney, Australia. The robot was deployed inside the two dewatered but internally cement-lined water pipes and run about $3 \mathrm{~m}$ and $5.3 \mathrm{~m}$ for the pipe 1 of $500 \mathrm{~mm}$ diameter and pipe 2 of 450 diameter, respectively. In each run, the robot internally scanned six lines along the pipe sections using its six mounted PEC sensors. It is noted that the sensor has a cross section area of $50 \mathrm{~mm}^{2}$; that is, a single PEC sensor can capture an average RWT measurement under its footprint of $50 \mathrm{~mm} \times 50 \mathrm{~mm}$, and each scanned line has a width of $50 \mathrm{~mm}$. Due to the cement linings and diameters of the water pipes, the robot was required to run 5 times in each pipe to fully scan their internal surfaces. In that case, there are two overlapped scanned lines between the first and last runs in the pipe 2 .

It is noted that though the verification was conducted in the cement-lined cast iron pipes, our proposed approach can be applied for any type of water pipes as long as there exists spatial correlation in the sensor measurements. More importantly, our method is independent from quality of sensor measurements; hence, it can be similarly applied for different RWT data obtained by different NDE sensors.

Therefore, in order to verify the proposed approach, we firstly measured RWT at every location along the whole pipes. Those measurements are demonstrated in Figures 5a and 6a for the pipe 1 and pipe 2, respectively. For the simplicity purpose, we considered these measurements as the complete data sets. For each pipe example, if we assume the sensors can only sense a part of those measurements as shown in Fig. 3, the RWT predictions at the white areas in Fig. 3 are expected to be highly comparable to the RWT measurements (in the complete data set) at the same locations.

Now we assume that the robot inspected the water pipes by only one run on each pipe, e.g. as illustrated in Fig. 3. That is, there are approximately 20 percent and 21 percent of the RWT data on the pipe 1 and pipe 2, respectively, gathered by the sensors in one run of the robot. We expect to build a 3D GP model for the RWT data on each pipe based on the RWT measurements, and then employ the learned models to correspondingly predict RWT at the remaining areas where the PEC sensors did not measure.

To be fair in the verification process, we utilized the crossvalidation method. In equivalent words, we assumed that the PEC sensors could measure RWT at any random lines of locations as long as they cover 20 percent or 21 percent of the pipe internal surface area for the pipe 1 and pipe 2, respectively, then we used the RWT measurements to predict the counterpart values at the unmeasured areas.

As previously mentioned, each water pipe requires five runs of the robot to fully sense RWT on the whole pipe surface, hence we designed five tests circumferentially presenting the five different starting location of the first PEC sensor. It is noted that since the six PEC sensors were equidistantly designed and fabricated along the circumference, once the first sensor started at a new location, the others would shift to the other locations accordingly. The locations of the first PEC sensor in the five tests were arranged as below.

- Test 1: The first sensor started at the crown of the pipe.

- Test 2: The first sensor started at 12 degrees from the crown of the pipe.

- Test 3: The first sensor started at 24 degrees from the crown of the pipe.

- Test 4: The first sensor started at 36 degrees from the crown of the pipe.

- Test 5: The first sensor started at 48 degrees from the crown of the pipe.

\section{A. RWT Prediction on Big Corrosion Patch Water Pipe - Pipe 1}

In the first pipe, the RWT measurements on the whole pipe collected by the sensors (also considered as a complete data set) as illustrated in Fig. 5a shows that the pipe wall has the quite big corrosion patches. In that case, the spatial correlation of RWT is quite large. In the intuitive examples (i.e. Test 1) as shown in Fig. 5, it was assumed that the RWT measurements after one robot run along the pipe as depicted by the six 50 $\mathrm{mm}$ width coloured lines in Fig. 3a was utilized to train a 3D GP model of RWT on the whole pipe, which was then employed to infer RWT values at the remaining unmeasured areas (i.e. the white areas in Fig. 3a).

As previously discussed in Section III, there are three popular marginal distributions including Gumbel, Weibull and GM that can be exploited to marginalize RWT measurements and convert them to the standard normally distributed data for training the 3D GP model. That is, we had the three separately learned 3D GP models corresponding to the three differently converted standard normally distributed data sets. As a result, we conducted the predictions of RWT values at uninspected areas based on the three separately learned 3D GP models and filled up the white areas as demonstrated in Fig. 3a, respectively. The fully reconstructed RWT data in Test 1 comprising the RWT measurements and predictions on the full pipe corresponding to separately learned 3D GP models (and their marginal distribution counterparts of Gumbel, Weibull and GM) are demonstrated in Figures 5b, 5c and 5d, respectively. Qualitatively, the fully reconstructed RWT data as shown in Figures 5b, 5c and 5d are highly comparable with the complete data set as depicted in Fig. 5a though only 20 percent of the complete data set were collected and used in the model training.

Quantitatively, we computed the root mean square errors (RMSEs) between the RWT predictions and their corresponding measurements in the complete data set at the same assumingly unmeasured locations (in the cross-validation method) and summarized the results in all the five different tests in Table II for the three models, respectively. Overall, the RMSE results in Table II show that the predictions are highly acceptable. It is noted that the second column of Table II are the results of Test 1, whose predictions are illustrated in Fig. 5 .

More importantly, one of the important information from RWT data is its minimum value, which may help water utilities 


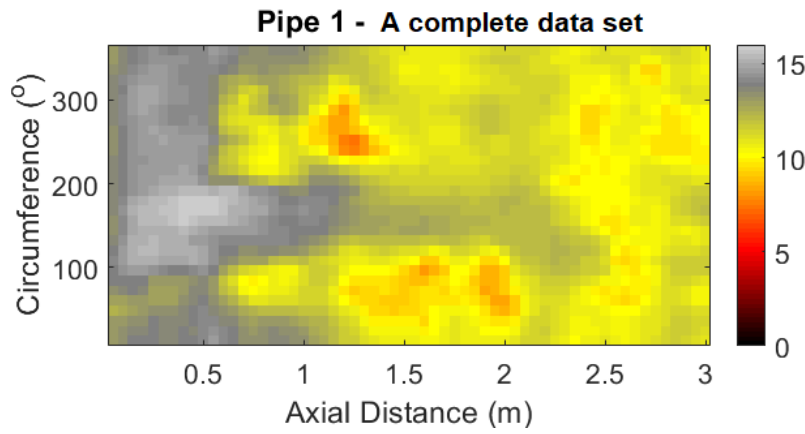

(a)

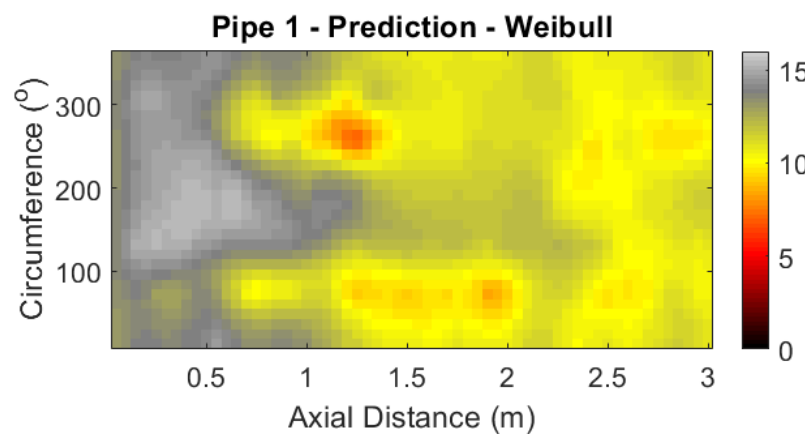

(c)

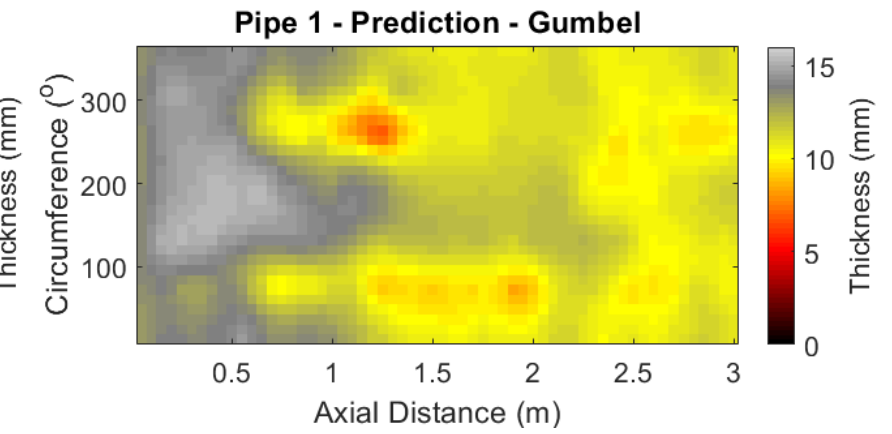

(b)

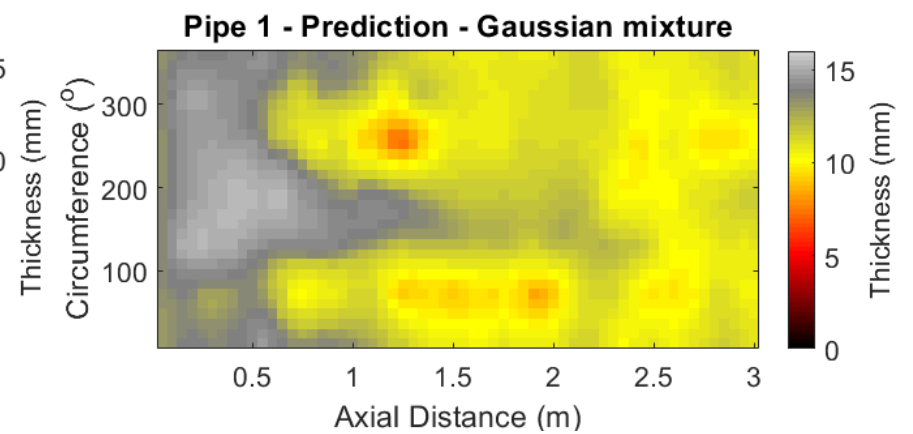

(d)

Fig. 5: Remaining wall thickness maps (collected and predicted data) of the pipe 1: (a) a complete data set, (b) fully reconstructed data based on Gumbel, (c) fully reconstructed data based on Weibull, (d) fully reconstructed data based on Gaussian mixture.

TABLE II: COMPARISONS OF RMSEs (mm) OF THE PREDICTED DATA AT THE UNMEASURED LOCATIONS ON PIPE 1 WITH RESPECT TO THE CORRESPONDING MEASUREMENTS IN THE COMPLETE DATA SET AMONG THREE MODELS

\begin{tabular}{|l|l|l|l|l|l|}
\hline Marginal distribution & Test 1 & Test 2 & Test 3 & Test 4 & Test 5 \\
\hline Gumbel & 0.539 & 0.530 & 0.437 & 0.461 & 0.469 \\
Weibull & 0.520 & 0.521 & 0.444 & 0.470 & 0.472 \\
Gaussian mixture & 0.521 & 0.476 & 0.483 & 0.529 & 0.523 \\
\hline
\end{tabular}

take necessary action to prevent a water pipe from failures. If the pipe can be fully inspected, the minimum RWT value is apparently obtained by the sensing. Nevertheless, in our proposed approach, since the PEC sensors only measured 20 percent of the full pipe, we validated the minimum RWT value obtained by the fully reconstructed RWT data, where the results for the five tests computed by the three models are summarized in Table III. It is noted that the real minimum RWT value obtained by the sensors on the pipe 1 is $7.35 \mathrm{~mm}$. It can be clearly seen that in Test 1 and 5 , the predictions of the minimum RWT value on the pipe 1 are highly accurate as compared with its measurement regardless the marginal distributions. In Tests 2, 3 and 4, the predicted results are still practically acceptable with a maximum absolute error of about $1 \mathrm{~mm}$.

Overall, the RWT results obtained by the proposed method are acceptable in practice while it can save about 80 percent of the inspection time as compared to the full inspection.
TABLE III: COMPARISONS OF THE MINIMUM THICKNESSES (mm) OF THE FULLY RECONSTRUCTED DATA ON PIPE 1 WITH RESPECT TO THE MEASUREMENT IN THE COMPLETE DATA SET AMONG THREE MODELS

\begin{tabular}{|l|l|l|l|l|l|}
\hline Marginal distribution & Test 1 & Test 2 & Test 3 & Test 4 & Test 5 \\
\hline Gumbel & 6.991 & 8.350 & 8.141 & 8.083 & 7.515 \\
Weibull & 7.232 & 8.350 & 8.225 & 8.150 & 7.600 \\
Gaussian mixture & 7.350 & 8.350 & 8.225 & 8.150 & 7.600 \\
\hline
\end{tabular}

\section{B. RWT Prediction on Small Corrosion Patch Water Pipe - Pipe 2}

In order to verify how the proposed approach performs on the corroded water pipe whose wall has smaller corrosion patches, we conducted the experiments on the pipe 2 with the RWT complete data set shown in Fig. 6a. Since the corroded pipe wall has the different geometry at the inspecting time, the characteristics of the spatial correlation in RWT of the pipe was believed to differ from those in the pipe 1 , which causes influences on the proposed technique.

We also conducted five tests whose designs were previously mentioned; and the RWT measurements collected in one robot run for Test 1 are shown by the six $50 \mathrm{~mm}$ width coloured lines in Fig. 3b. Similar to the pipe 1, the three separate 3D GP models were built based on the three marginal distributions and the measured RWT data in each test. The predicted results were used to fill up the white areas, i.e. the unmeasured locations, in Fig. 3b; and the fully reconstructed RWT data corresponding to the three models in Test 1 are demonstrated in Figures 6b, 6c and 6d, respectively. 


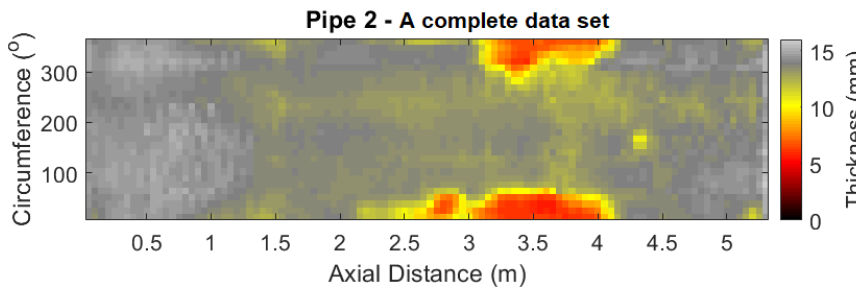

(a)

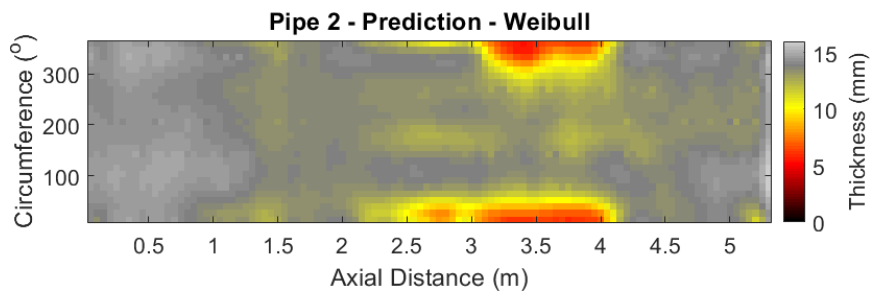

(c)

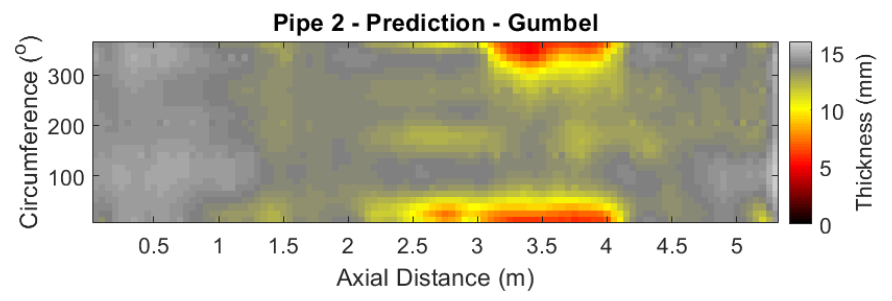

(b)

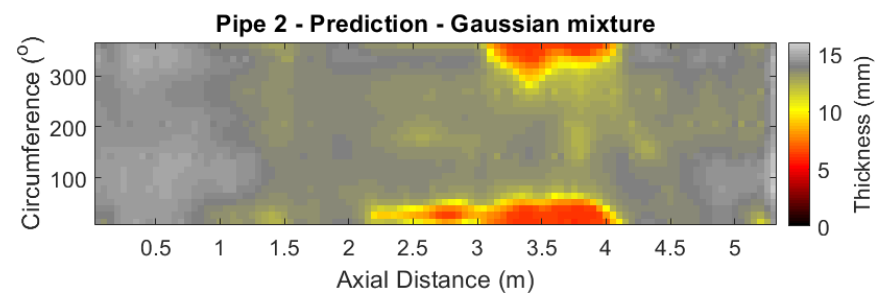

(d)

Fig. 6: Remaining wall thickness maps (collected and predicted data) of the pipe 2: (a) a complete data set, (b) fully reconstructed data based on Gumbel, (c) fully reconstructed data based on Weibull, (d) fully reconstructed data based on Gaussian mixture.

TABLE IV: COMPARISONS OF RMSEs (mm) OF THE PREDICTED DATA AT THE UNMEASURED LOCATIONS ON PIPE 2 WITH RESPECT TO THE CORRESPONDING MEASUREMENTS IN THE COMPLETE DATA SET AMONG THREE MODELS

\begin{tabular}{|l|l|l|l|l|l|}
\hline Marginal distribution & Test 1 & Test 2 & Test 3 & Test 4 & Test 5 \\
\hline Gumbel & 0.596 & 0.527 & 0.558 & 0.602 & 0.551 \\
Weibull & 0.567 & 0.515 & 0.591 & 0.624 & 0.537 \\
Gaussian mixture & 0.468 & 0.546 & 0.591 & 0.641 & 0.475 \\
\hline
\end{tabular}

Undoubtedly, the RWT predictions at 79 percent of the pipe wall based on 21 percent of the RWT measurements are presenting highly comparably to their corresponding RWT measurements in the complete data set, which can be apparently seen in Fig. 6 for Test 1 .

Furthermore, the RMSEs of the predicted RWT values as compared with the corresponding measurements in the complete data set in the five tests were calculated and summarized in Table IV. It can be seen that these errors are quite consistent regardless where the PEC sensors collected the data. More interestingly, by comparing the RMSEs in both Tables II and IV, these results are quite similar though two pipes have been buried in the faraway locations from each other and they have the different geometries and corrosion processes. That indicates the consistency in the prediction accuracy of the proposed approach at a particular percentage of inspection. Furthermore, in Section III it stated that the GM fits the RWT measurements in Test 1 of the pipe 2 better than the other two counterparts, which consequently leads to a smaller RMSE in the predictions as shown in the second column of Table IV.

Regarding the minimum RWT value obtained by our proposed prediction technique as compared with the real measurement obtained by the sensors for the pipe 2, which is $5.56 \mathrm{~mm}$, in contrast to the results in the pipe 1, those in all the five tests summarised in Table V reveal that the method consistently predicts the minimum RWT value on the pipe
TABLE V: COMPARISONS OF THE MINIMUM THICKNESSES (mm) OF THE FULLY RECONSTRUCTED DATA ON PIPE 2 WITH RESPECT TO THE MEASUREMENT IN THE COMPLETE DATA SET AMONG THREE MODELS

\begin{tabular}{|l|l|l|l|l|l|}
\hline Marginal distribution & Test 1 & Test 2 & Test 3 & Test 4 & Test 5 \\
\hline Gumbel & 5.195 & 5.075 & 5.463 & 5.919 & 4.633 \\
Weibull & 5.491 & 5.364 & 5.564 & 6.003 & 5.186 \\
Gaussian mixture & 5.911 & 5.665 & 5.564 & 6.193 & 5.911 \\
\hline
\end{tabular}

very well despite the fact that the 21 percent of the RWT data utilized for training the 3D GP models were gathered from the different if not random locations along the pipe.

\section{CONCLUSIONS}

A new data prediction approach to effectively predict RWT of in-service water pipes has been proposed and discussed in this paper. The proposed method only requires the sensors to measure RWT on a part of the total water pipe surface area and then utilizes the measurements to predict RWT in the remaining area. To effectively predict RWT, the nonGaussian measurements are first converted to the standard normally distributed data by a marginal distribution such as Gumbel, Weibull and GM, and the converted data is then fed into a 3D GP model to present RWT in the whole pipe. The spatial correlation of the data allows the model to predict RWT at any unmeasured locations on the pipe. Both the RWT measurements and predictions can be merged to fully reconstruct the RWT data on the whole pipeline. The implementations of our method in two realistic corroded water pipes with the promising results have indicated its practicality.

In this work, although we employed the RWT measurements obtained by the PEC sensors to evaluate our method, the proposed algorithm can be effectively applied for different RWT data gathered by different NDE techniques such as the remote field eddy current. Moreover, since the proposed approach can fully reconstruct RWT data of a water pipe, it 
can be used to either predict maximum loss of section or infer pit depth and conformation on the pipe. Nevertheless, it is noted that considering measurement uncertainties is beyond our work; and though the predictions have been shown to be highly comparable with the corresponding sensor measurements, prediction uncertainty comprising model uncertainty and measurement error may not be trivial if a sensor is inaccurate.

Furthermore, though in the demonstrations about 20 percent of the data are used to reconstruct the whole data set, the proposed method can be generally applied for different percentages of the data (e.g. 10 percent or 30 percent). However, using 10 percent of the data for reconstructing the whole data set leads to higher prediction uncertainties than using 20 percent. In other words, the more measurements can be obtained by sensors, the more accurate predicted RWT values are.

\section{REFERENCES}

[1] J. Valls Miro, J. Rajalingam, T. Vidal-Calleja, F. Bruijn, T. Wood, D. Vitanage, N. Ulapane, B. Wijerathna, and D. Su, "A live test-bed for the advancement of condition assessment and failure prediction research on critical pipes," Water Asset Management International, vol. 10(2), pp. 03-08, 2014.

[2] A. Rainer, T. F. Capell, N. Clay-Michael, M. Demetriou, T. S. Evans, D. A. Jesson, M. J. Mulheron, L. Scudder, and P. A. Smith, "What does NDE need to achieve for cast iron pipe networks?" Infrastructure Asset Management, vol. 4, no. 2, pp. 68-82, 2017.

[3] R. B. Petersen, T. Wells, and R. E. Melchers, "Development of long-term localised corrosion of cast iron pipes in backfill soils based on time of wetness," Corrosion Engineering, Science and Technology, vol. 0, no. 0, pp. $1-12,2020$.

[4] D. Vitanage, J. Kodikara, and G. Allen, "Collaborative research on condition assessment and pipe failure prediction for critical water mains," Water Asset Management International, vol. 10, pp. 15-18, 2014.

[5] D. Hunt, M. Hussein, C. Stewart, G. Dissanayake, J. Valls Miro, J. Olson, and R. Rossi, "Rapid response non-destructive inspection robot for condition assessment of critical water mains," in Proc. Australian Conference on Robotics and Automation, Canterbury, New Zealand, December 2018, pp. 1-7.

[6] J. Kodikara, S. Rathnayaka, J. Zhang, C. Crawly, D. Zhang, and F. Blaha, "Lessons learned from large-diameter pipe failure case studies," in Proc. Conferece on Pipelines, Arizona, USA, August 2017, pp. 561-571.

[7] W. Wang, A. Zhou, G. Fu, C. Q. Li, D. Robert, and M. Mahmoodian, "Evaluation of stress intensity factor for cast iron pipes with sharp corrosion pits," Engineering Failure Analysis, vol. 81, pp. 254-269, 2017.

[8] A. Fahimi, T. S. Evans, J. Farrow, D. A. Jesson, M. J. Mulheron, and P. A. Smith, "On the residual strength of aging cast iron trunk mains: Physically-based models for asset failure," Materials Science \& Engineering A, vol. 663, pp. 204-212, 2016.

[9] J. Ji, D. J. Robert, C. Zhang, D. Zhang, and J. Kodikara, "Probabilistic physical modelling of corroded cast iron pipes for lifetime prediction," Structural Safety, vol. 64, pp. 62-75, 2017.

[10] G. Ugoh, R. Cunningham, J. Farrow, M. Mulheron, and D. Jesson, "On the residual strength of ageing cast iron wastewater assets: Models for failure," Materials Science and Engineering: A, vol. 768, p. 138221, 2019.

[11] M. Mokhtari and R. E. Melchers, "Reliability of the conventional approach for stress/fatigue analysis of pitting corroded pipelines development of a safer approach," Structural Safety, vol. 85, p. 101943 , 2020.
[12] Z. Liu and Y. Kleiner, "State of the art review of inspection technologies for condition assessment of water pipes," Measurement, vol. 46(1), pp. 1-15, 2013.

[13] L. Nguyen, N. Ulapane, J. V. Miro, G. Dissanayake, and F. Munoz, "Improved signal interpretation for cast iron thickness assessment based on pulsed eddy current sensing," in Proc. IEEE Conference on Industrial Electronics and Applications, Siem Reap, Cambodia, June 2017, pp. 2005-2010.

[14] N. Ulapane, L. Nguyen, J. V. Miro, A. Alempijevic, and G. Dissanayake, "Designing a pulsed eddy current sensing set-up for cast iron thickness assessment," in Proc. IEEE Conference on Industrial Electronics and Applications, Siem Reap, Cambodia, June 2017, pp. 901-906.

[15] N. Ulapane, L. Nguyen, J. V. Miro, and G. Dissanayake, "A solution to the inverse pulsed eddy current problem enabling 3d profiling," in Proc. IEEE Conference on Industrial Electronics and Applications, Wuhan, China, June 2018, pp. 1267-1272.

[16] N. Ulapane, A. Alempijevic, J. Valls Miro, and T. Vidal-Calleja, "Nondestructive evaluation of ferromagnetic material thickness using pulsed eddy current sensor detector coil voltage decay rate," NDT \& E International, vol. 100, pp. $108-114,2018$.

[17] N. Ulapane and L. Nguyen, "Review of pulsed-eddy-current signal feature-extraction methods for conductive ferromagnetic materialthickness quantification," Electronics, vol. 8, pp. 1-13, 2019.

[18] G. Y. Tian and A. Sophian, "Reduction of lift-off effects for pulsed eddy current NDT," NDT \& E International, vol. 38, no. 4, pp. 319 - 324 2005.

[19] G. Y. Tian, Y. Li, and C. Mandache, "Study of lift-off invariance for pulsed eddy-current signals," IEEE Transactions on Magnetics, vol. 45, no. 1, pp. 184-191, 2009.

[20] M. Ricci, G. Silipigni, L. Ferrigno, M. Laracca, I. D. Adewale, and G. Y. Tian, "Evaluation of the lift-off robustness of eddy current imaging techniques," NDT \& E International, vol. 85, pp. 43 - 52, 2017.

[21] L. Nguyen and J. Valls Miro, "An efficient 3-dimensional model for remaining wall thicknesses of cast iron pipes in non-destructive testing," IEEE Sensor Letters, vol. 4(7), p. 6001104, 2020.

[22] L. V. Nguyen, S. Kodagoda, and R. Ranasinghe, "Spatial sensor selection via Gaussian Markov random fields," IEEE Transaction on Systems, Man, and Cybernetics: Systems, vol. 46(9), pp. 1226-1239, 2016

[23] Z. S. Asadi and R. E. Melchers, "Extreme value statistics for pitting corrosion of old underground cast iron pipes," Reliability Engineering and System Safety, vol. 162, pp. 64-71, 2017.

[24] D. Benstock and F. Cegla, "Extreme value analysis (EVA) of inspection data and its uncertainties," NDT\&E International, vol. 87, pp. 68-77, 2017.

[25] L. Nguyen, J. Valls Miro, L. Shi, and T. Vidal-Calleja, "Gaussian mixture marginal distributions for modelling remaining pipe wall thickness of critical water mains in non-destructive evaluation," in Proc. IEEE International Conference on Cybernetics and Intelligent Systems (CIS) and IEEE Conference on Robotics, Automation and Mechatronics (RAM), Bangkok, Thailand, November 2019, pp. 257-262.

[26] C. Zhang, S. Rathnayaka, B. Shannon, J. Ji, and J. Kodikara, "Numerical interpretation of pressurized corroded cast iron pipe tests," International Journal of Mechanical Sciences, vol. 128-129, pp. 116-124, 2017.

[27] C. Zhang, J. Ji, J. Kodikara, and B. Rajani, "Hyperbolic constitutive model to study cast iron pipes in 3-d nonlinear finite element analyses," Engineering Failure Analysis, vol. 75, pp. 26-36, 2017.

[28] C. E. Rasmussen and C. K. I. Williams, Gaussian processes for machine learning. The MIT Press, Cambridge, Massachusetts, London, England, 2006.

[29] H. Zhu and L. M. Zhang, "Characterizing geotechnical anisotropic spatial variations using random field theory," Canadian Geotechnical Journal, vol. 50, pp. 723-734, 2013.

[30] L. Nguyen, S. Kodagoda, R. Ranasinghe, and G. Dissanayake, "Information-driven adaptive sampling strategy for mobile robotic wireless sensor network," IEEE Transaction on Control Systems Technology, vol. 24(1), pp. 372-379, 2016 